\title{
ANÁLISE NUMÉRICA DO ESTADO DE DANIFICAÇÃO DE ESTRUTURAS DE CONCRETO ARMADO CONDICIONADAS À CORROSÃO UNIFORME
}

\author{
FELIX, EMERSON FELIPE \\ Doutorando em Eng. Civil/Estruturas \\ USP/SET/EESC \\ São Carlos/SP; Brasil \\ emerson.felipe.felix@gmail.com
}

\author{
CARRAZEDO, ROGÉRIO \\ Professor Dr. em Eng. Civil/Estruturas \\ USP/SET/EESC \\ São Carlos/SP; Brasil \\ rogcarrazedo@sc.usp.br
}

\section{RESUMO}

A fim de controlar e minimizar os efeitos impostos pela corrosão, faz-se necessário deter ferramentas capazes de modelála e, assim, avaliar o estado de danificação do concreto de cobrimento ao longa da vida útil do elemento estrutural. Sabendo disso, este trabalho apresenta uma avaliação da evolução do dano em vigas de concreto armado quando submetidas a diferentes ambientes e, com concretos produzidos com distintos traços. A simulação da corrosão e de seus efeitos é feita por meio de um modelo que foi desenvolvido baseando-se no Método dos Elementos Finitos Posicional, e que considera as não-linearidades física e geométrica. Os resultados encontrados demonstram a eficiência da formulação desenvolvida e apontam a aplicabilidade da mesma para a modelagem e simulação dos efeitos deteriorativos da corrosão no concreto armado. Ademais, os resultados encontrados neste trabalho mostram que a depender da configuração da disposição das armaduras, do cobrimento, do traço do concreto e do ambiente de exposição, o nível de danificação de um elemento de concreto pode ser significativamente elevado, comprometendo a sua integridade estrutural e, por consequência, a sua vida útil.

Palavras-chave: concreto armado, mecânica do dano, degradação do concreto, corrosão.

\begin{abstract}
In order to control and minimize the effects imposed by corrosion, it is necessary to hold tools able to model it and thereby assess the state of damage of the cover concrete to the long life of the structural element. Knowing this, this paper presents an assessment of the damage evolution in reinforced concrete beams when subjected to different environments and with concrete produced with distinctive features. The simulation of corrosion and its effects is made by a model that was developed based on the Positional Finite Element Method, which considers the physical and geometric nonlinearities. The results show the efficiency of the developed formulation and point out its applicability to the modeling and simulation of the deteriorating effects of corrosion in reinforced concrete. Moreover, the results found in this work show that depending on the configuration of the reinforcement arrangement, the covering, the concrete composition and the exposure environment, the level of concrete damage can be significantly high, which can compromise its structural integrity and therefore its useful life.
\end{abstract}

Keywords: reinforced concrete, damage mechanics, concrete deterioration, corrosion.

\section{INTRODUÇÃO}

Uma das principais causas de redução da vida útil de estruturas de concreto armado é a corrosão, uma vez que esta envolve a perda de material da superfície do aço como resultado de uma ação química, e a perda material leva a redução de área efetiva na seção transversal e consequentemente a diminuição da capacidade de suportar cargas (KARI; PUTTONEN; SKANTZ, 2014). No Brasil, por exemplo, a corrosão possuí um índice de ocorrência em estruturas de concreto que varia de 14 a 64\% a depender da região de análise (CARMONA; MAREGA, 1988; DAL MOLIN, 1988; ANDRADE, 1992).

Entretanto a corrosão pode ser evitada a partir de uma determinada camada de concreto (cobrimento) entre o aço e o ambiente externo. Isso ocorre devido a alcalinidade do concreto, enquanto os metais corroem em meio ácido, o meio alcalino os protegem. 
A alcalinidade do concreto se dá devido aos poros microscópicos que contêm altas concentrações de óxidos de cálcio, sódio e potássio solúveis. Estes formam hidróxidos, alcalinos na presença de água, criando uma condição de $\mathrm{pH}$ ótimo (entre 12 e 13). Deste modo o concreto protege o aço da corrosão tanto fisicamente formando uma camada protetora para a armadura, quanto quimicamente por meio da condição alcalina que induz a formação de uma película passiva, na superfície do aço, muito densa, fina e de óxido, levando a uma baixa taxa de corrosão (BROOMFIELD, 2007; KÖLIÖ et al., 2017).

A modelagem da deterioração por corrosão tem utilizado os processos fenomenológicos em que a corrosão do aço no concreto armado é segmentada e resumida em dois estágios, o de iniciação e o de propagação da corrosão (TUUTTI, 1982; BAKKER, 1988; RAO et al., 2017).

A fase de iniciação se caracteriza pela difusão de diferentes agentes agressivos presentes no meio ambiente, a exemplo, o dióxido de carbono $\left(\mathrm{CO}_{2}\right)$. O gás carbônico penetra na matriz porosa do concreto reagindo com os hidróxidos de cálcio $\left(\mathrm{Ca}(\mathrm{OH})_{2}\right)$ presente na pasta de cimento, levando a formação do carbonato de cálcio $\left(\mathrm{CaCO}_{3}\right)$. Na literatura este processo recebe o nome de carbonatação. A reação de carbonatação começa na superfície do concreto progredindo internamente com o tempo. À medida que a frente de carbonatação atinge a profundidade do aço, inicia-se a fase de progressão da corrosão, não havendo a ocorrência de nenhum dano real à estrutura até este ponto (TUUTTI, 1982; POSSAN, 2010; KÖLIÖ et al., 2017).

A propagação é determinada pela taxa de corrosão (governada pela disponibilidade de oxigênio, umidade relativa e temperatura) e a capacidade da cobertura do concreto para suportar tensões internas. O óxido férrico não hidratado $\left(\mathrm{Fe}_{2} \mathrm{O}_{3}\right)$ tem um volume de cerca de duas vezes o do aço que substitui quando totalmente denso. O óxido férrico ao hidratar-se, se expande ainda mais tornando-se poroso, aumentando o volume na interface aço-concreto de três a dez vezes, causando a perda de área efetiva de aço. Essa expansão leva à fissuração, que ocorre quando as tensões induzidas pela camada crescente de produtos de corrosão excedem a capacidade de tração do concreto, especialmente em estruturas onde o aço é colocado relativamente perto da superfície do concreto. A fissuração acelera a penetração de agentes nocivos e pode causar maiores danos, por exemplo, o lascamento do concreto de cobrimento (Figura 1), onde todo um segmento da cobertura de concreto se desprende da superfície da estrutura (BROOMFIELD, 2007; KÖLIÖ et al., 2017).
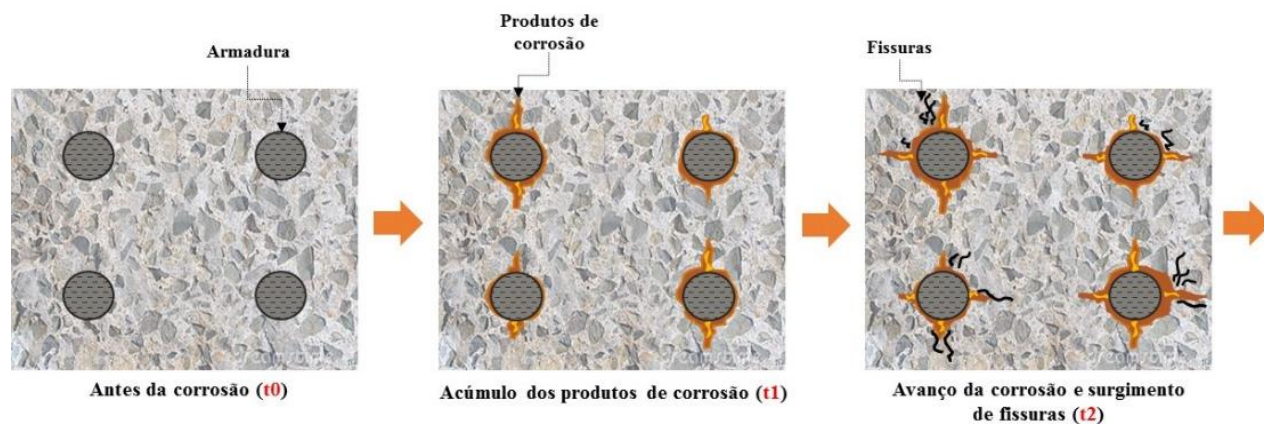

Figura 1: Estágios dos danos causados no concreto no período de progressão da corrosão.

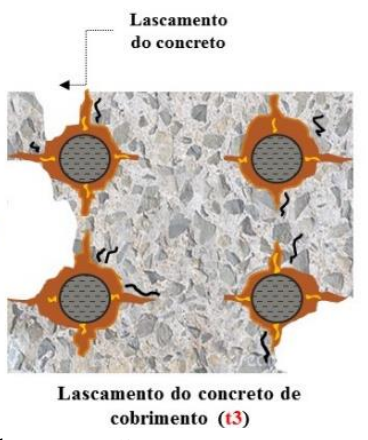

Diante do exposto, apresenta-se neste trabalho um modelo numérico para a representação da expansão do concreto armado devido à formação de produtos de corrosão. A formulação é baseada no Método dos Elementos Finitos Posicional (MEFP), desenvolvida em nível mesoscópico, que tem por finalidade a análise não-linear geométrica de estruturas bidimensionais constituídas de materiais compósitos particulados. Para a representação da não linearidade física do material, decidiu-se por acoplar à formulação o modelo de dano de Mazars, devido a sua simplicidade e pelo fato de se utilizar uma grandeza escalar para representar a danificação do material.

\section{EXPANSÃO DO CONCRETO DEVIDO AOS PRODUTOS DE CORROSÃO: MODELAGEM}

Com o intuito em modelar a expansão do concreto armado decorrente da corrosão, implementou-se inicialmente um programa em linguagem Fortran para a análise estrutural não-linear geométrica de sólidos bidimensionais compósitos com partículas (para a representação do concreto armado) baseado no Método dos Elementos Finitos Posicional, descrito em Coda (2003) e adaptado em Paccola e Coda (2016). Em seguida realizou-se uma adaptação no programa para que o mesmo incorporasse a não-linearidade física do material, fazendo o uso da mecânica do dano, especificamente, do modelo 
de dano de Mazars. Por fim, acoplou-se ao código a formulação desenvolvida por Kiani e Shodja (2011) para a representação da formação dos produtos de corrosão.

\subsection{Modelo de Dano de Mazars}

A mecânica do dano tem por função descrever a evolução dos fenômenos de perda de resistência e rigidez evolutiva de um material, representando o material desde o seu estado íntegro até o instante de ruptura, o estado final. Para isso, a mecânica do dano fundamenta-se nos princípios, e métodos, da mecânica do contínuo e da termodinâmica dos processos irreversíveis.

De acordo com Proença (1992) o dano não é uma grandeza física diretamente mensurável, porém, para fins da modelagem matemática, é possível relacioná-lo com a redução progressiva de características mecânicas, por exemplo, o módulo de elasticidade. Deste modo, uma variável interna representativa do estado de deterioração do material é definida de forma a permitir quantificar e distinguir, macroscopicamente, um solido deteriorado, de outro íntegro.

O modelo constitutivo proposto por Mazars (1984), para o caso de carregamento monótono crescente, admite as seguintes hipóteses:

- O concreto em processo de dano evolutivo apresenta um comportamento elástico, desprezando assim, deformações permanentes evidenciadas em situações de descarregamento;

- Localmente o dano é causado pela presença de extensões, pelo menos na direção de uma das deformações principais;

- O dano é representado por uma variável escalar (D), cuja evolução ocorre ao atingir um critério de resistência.

O estado de alongamento do material é caracterizado pela deformação equivalente $\tilde{\varepsilon}$, conforme apresenta a Equação (1). Todo o equacionamento a seguir foi escrito de acordo com Proença (1992).

$$
\begin{gathered}
\tilde{\varepsilon}=\sqrt{\left\langle\varepsilon_{1}\right\rangle_{+}^{2}+\left\langle\varepsilon_{2}\right\rangle_{+}^{2}+\left\langle\varepsilon_{3}\right\rangle_{+}^{2}} \\
\left\langle\varepsilon_{i}\right\rangle_{+}=\frac{1}{2}\left[\varepsilon_{i}+\left|\varepsilon_{i}\right|\right]
\end{gathered}
$$

em que $\varepsilon_{i}$ é a deformação principal na direção $i$.

Segundo o critério adotado, o processo de dano inicia quando $\tilde{\varepsilon}$ atinge um valor convencional igual à deformação $\varepsilon_{d 0}$, correspondente ao esforço máximo em um ensaio de tração uniaxial. Para um estado geral de dano $D$, o critério que determina sua evolução é expresso pela Equação (3), sendo a lei de evolução do dano $D$, dada pelas Equações (4-5).

$$
\begin{gathered}
f(\tilde{\varepsilon}, D)=\tilde{\varepsilon}-S(D)=0, \operatorname{com} S(0)=\varepsilon_{d 0} \\
\dot{D}=0 \text { se } f<0 \text { ou } f=0 \text { e } \dot{f}<0 \\
\dot{D}=F(\tilde{\varepsilon})\langle\tilde{\varepsilon}\rangle_{+} \text {se } f=0 \text { e } \dot{f}=0
\end{gathered}
$$

em que $F(\tilde{\varepsilon})$ é uma função contínua e positiva da deformação equivalente $\tilde{\varepsilon}$ (de modo que $\dot{D} \geq 0 \forall \tilde{\varepsilon}$ ).

Nos casos particulares de tração e compressão uniaxial, a Equação (5) é substituída pela Equações (6) e (7) respectivamente.

$$
\begin{aligned}
& \dot{D}_{T}=F_{T}(\tilde{\varepsilon})\langle\tilde{\varepsilon}\rangle_{+} \text {se } f=0 \text { e } \dot{f}=0 \\
& \dot{D}_{C}=F_{C}(\tilde{\varepsilon})\langle\tilde{\varepsilon}\rangle_{+} \text {se } f=0 \text { e } \dot{f}=0
\end{aligned}
$$

No caso de solicitação monótona crescente as Equações (6) e (7) são integráveis, sendo possível escrever $D_{T}$ e $D_{C}$ de forma explicita conforme vê-se nas Equações (8) e (9). 


$$
\begin{aligned}
& D_{T}(\tilde{\varepsilon})=1-\frac{\varepsilon_{d 0}\left(1-A_{T}\right)}{\tilde{\varepsilon}}-\frac{A_{T}}{e^{\left[B_{T}\left(\tilde{\varepsilon}-\varepsilon_{d 0}\right)\right]}} \\
& D_{C}(\tilde{\varepsilon})=1-\frac{\varepsilon_{d 0}\left(1-A_{C}\right)}{\tilde{\varepsilon}}-\frac{A_{C}}{e^{\left[B_{C}\left(\tilde{\varepsilon}-\varepsilon_{d 0}\right)\right]}}
\end{aligned}
$$

em que $A_{T}, A_{C}, B_{T}$ e $B_{C}$ são parâmetros do material a serem identificados com base em resultados de ensaios uniaxiais de tração e compressão.

No caso de estado de esforço multiaxial em um ponto, Mazars (1984) propôs para $D$ a relação expressa na Equação (10).

$$
\begin{gathered}
D=\alpha_{T} D_{T}+\alpha_{C} D_{C} \\
\alpha_{T}=\sum_{i} H_{i} \frac{\varepsilon_{T i}\left(\varepsilon_{T i}+\varepsilon_{C i}\right)}{\tilde{\varepsilon}^{2}} \\
\alpha_{C}=\sum_{i} H_{i} \frac{\varepsilon_{C i}\left(\varepsilon_{T i}+\varepsilon_{C i}\right)}{\tilde{\varepsilon}^{2}}
\end{gathered}
$$

em que os coeficientes $\alpha_{T} \alpha_{C}$ assumem valores entre 0 e 1 e são tais que a soma é sempre unitária, $H_{i}\left(\varepsilon_{i}\right)$ é a função degrau de Heaviside.

Finalmente, a lei constitutiva do material, definida no modelo de Mazars, válida na hipótese de carregamento monótono crescente é expressa na Equação (13).

$$
\sigma=\mathbb{D}(1-D) \varepsilon
$$

em que $\sigma$ representa o tensor de tensão e $\mathbb{D}$ é o tensor de rigidez elástico inicial.

\subsection{Modelo de formação dos produtos de corrosão}

Kiani e Shodja (2011) desenvolveram um modelo linear da formação dos produtos de corrosão em estruturas de concreto armado, utilizando dados experimentais referentes à corpos de prova cilíndricos de concreto contendo em seu centro uma armadura de diâmetro e propriedades conhecidas. A Figura 2 apresenta esquematicamente o modelo proposto pelos autores.

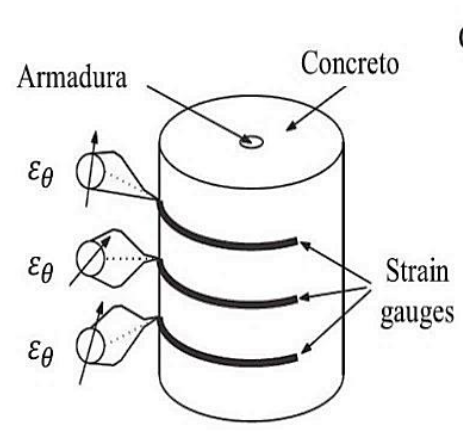

(a)

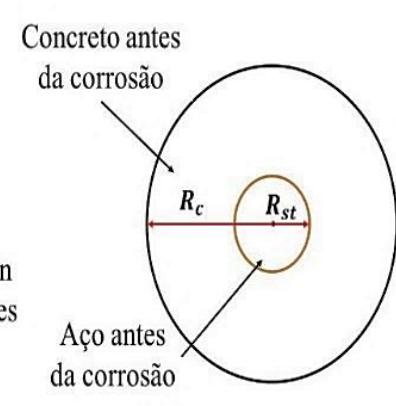

(b)

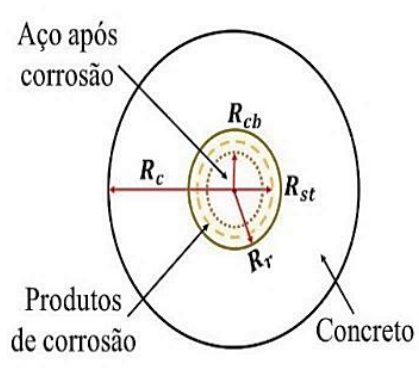

(c)

Figura 2: Representação do modelo linear da formação dos produtos de corrosão. Fonte: Felix (2018).

Na Figura 2(a) é apresentado um modelo dos corpos de prova utilizados nos ensaios em laboratório, na Figura 2(b) podese ver a seção transversal do concreto antes de iniciar o processo de corrosão. Com o desenvolvimento da corrosão os produtos são formados no entorno da armadura reduzindo o seu diâmetro, como mostra a Figura 2(c).

O modelo resume-se a determinação do raio residual de aço $\left(\mathrm{R}_{\mathrm{cb}}\right)$ e do raio da frente de avanço dos produtos de corrosão $\left(\mathrm{R}_{\mathrm{r}}\right)$, que podem ser obtidos respectivamente pelas Equações (14) e (15). Para a determinação destes parâmetros faz-se necessário calcular o volume gerado pelos produtos de corrosão Equação (16) e o volume de aço que as armaduras perderam Equação (17). 


$$
\begin{gathered}
R_{c b}=\sqrt{R_{s t}^{2}-\frac{V_{s}}{\pi}} \\
R_{r}=\sqrt{R_{c b}^{2}+\frac{V_{r}}{\pi}} \\
V_{r}(t)=\frac{\alpha}{\rho_{s t}} \sqrt{\int_{0}^{t} 2 \times 6,78 \times 10^{-10} \pi \cdot R_{s t} \cdot i_{c o r r} d t} \\
V_{s}(t)=\frac{r_{m}}{\rho_{s t}} \sqrt{\int_{0}^{t} 2 \times 6,78 \times 10^{-10} \pi \cdot R_{s t} \cdot i_{c o r r} d t}
\end{gathered}
$$

em que $R_{s t}$ refere-se ao raio inicial da armadura (m), $\alpha$ é a razão entre a densidade do aço e dos produtos de corrosão, $\rho_{s t}$ é a densidade do aço $\left(\mathrm{kg} / \mathrm{m}^{3}\right), i_{\text {corr }}$ é a densidade da corrente de corrosão $\left(\mathrm{A} / \mathrm{m}^{2}\right), r_{m}$ é a proporção de massa de ferro para a massa molecular dos produtos de corrosão e t (s) refere-se ao tempo de análise, sendo dado a partir do momento em que ocorre a despassivação da armadura.

\subsection{Acoplamento dos modelos}

A fim de apresentar o modelo final implementado, apresenta-se na Figura 3 um fluxograma referente ao acoplamento das três formulações, MEFP, dano de Mazars e o modelo de formação dos produtos de corrosão.

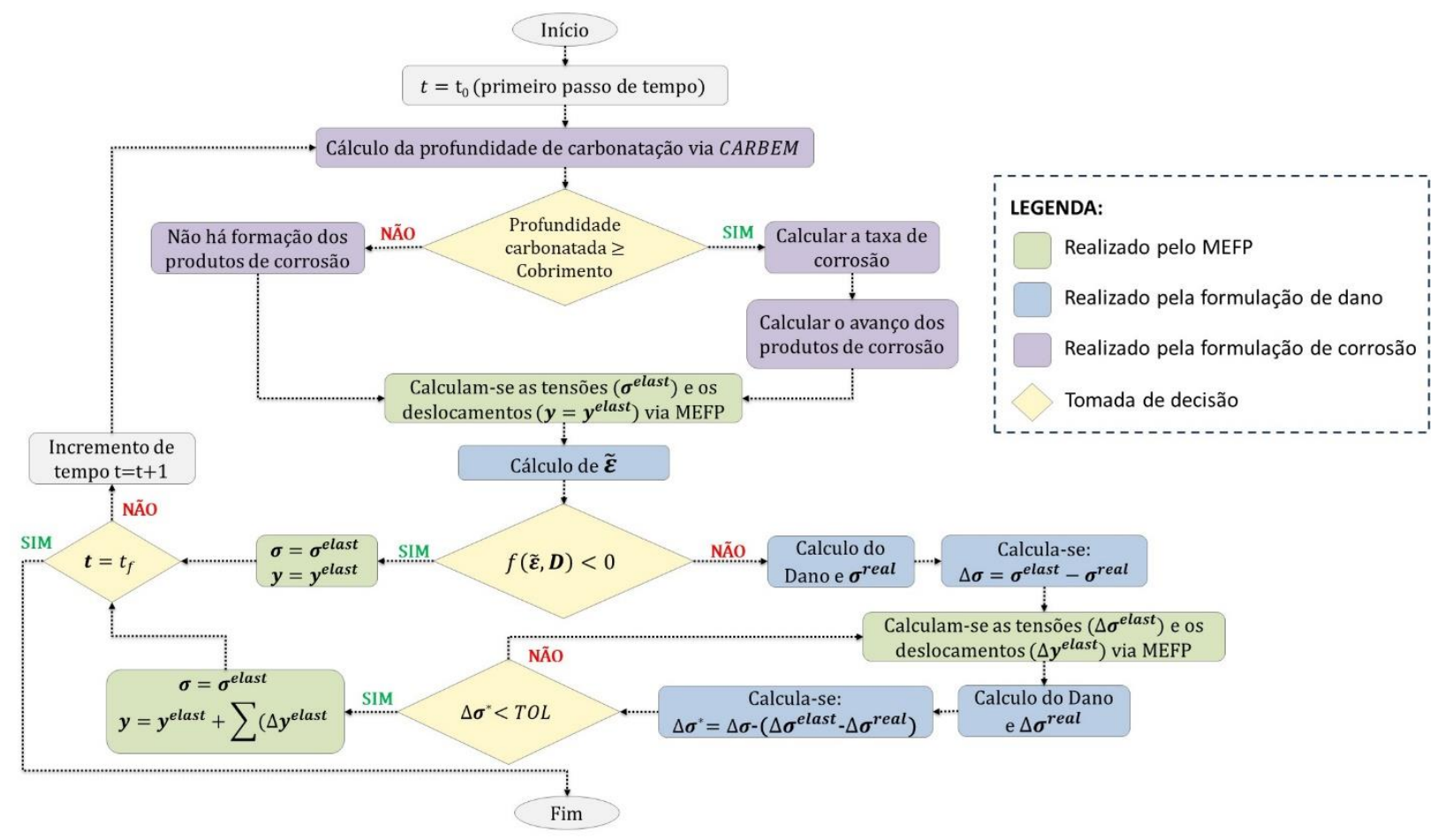

Figura 3: Fluxograma referente ao acoplamento dos modelos mecânicos e fenomenológico da corrosão.

\section{RESULTADOS}

Inicialmente, com o objetivo de comprovar a potencialidade do modelo desenvolvido, apresenta-se a validação dos acoplamentos dos modelos de dano e de formação dos produtos de corrosão. Em seguida é apresentado os resultados obtidos na modelagem da expansão causada pela corrosão em uma viga de concreto armado. 


\subsection{Validação do modelo de Mazars}

Para a validação do modelo de dano implementado, modelou-se uma viga parede de concreto e os resultados foram comparados com os obtidos por Proença, Papa e Maier (1991).

A estrutura consiste em uma viga parede com seção transversal retangular, de dimensão 20x50x270 cm. Na Figura 4 é apresentado um detalhamento simplificado da viga e o esquema referente ao carregamento imposto.

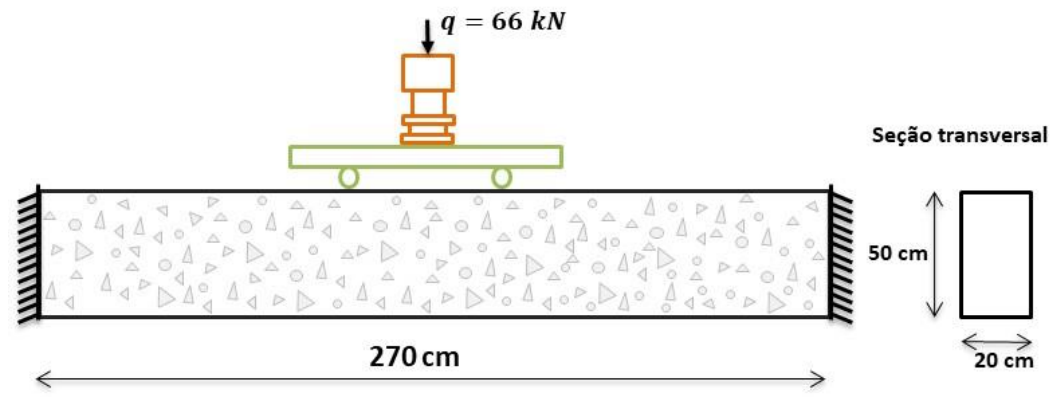

Figura 4: Esquematização da viga e carregamento modelado.

A discretização da malha de elementos finitos da viga parede de concreto armado foi feita com 2071 nós e 432 elementos triangulares com ordem de aproximação cúbica. A análise da viga parede foi realizada com controle de carga, com passos de 1 kN. Os dados referentes às propriedades dos materiais são apresentados na Tabela 1(a), enquanto que na Tabela 1(b) apresentam-se os dados referentes ao dano.

Tabela 1(a) - Propriedade dos materiais.

\begin{tabular}{c|c|c}
\hline Material & Propriedade & Valor \\
\hline \multirow{3}{*}{$\begin{array}{c}\text { Concreto } \\
(\text { C20 })\end{array}$} & Módulo de elasticidade $\left(\mathrm{kN} / \mathrm{cm}^{2}\right)$ & 2470,00 \\
\cline { 2 - 3 } & Coeficiente de Poisson & 0,20 \\
\cline { 2 - 3 } & Resistência à compressão $\left(\mathrm{kN} / \mathrm{cm}^{2}\right)$ & 2,00 \\
\cline { 2 - 3 } & Resistência à tração $\left(\mathrm{kN} / \mathrm{cm}^{2}\right)$ & 0,21 \\
\hline
\end{tabular}

Tabela 1(b) - Dados referentes ao dano.

\begin{tabular}{c|c}
\hline Propriedade & Valor \\
\hline$\varepsilon \mathrm{d} 0$ & $8,9 \mathrm{E}-5$ \\
\hline $\mathrm{A}_{\mathrm{T}}$ & 0,70 \\
\hline $\mathrm{B}_{\mathrm{T}}$ & 8000,00 \\
\hline $\mathrm{A}_{\mathrm{C}}$ & 1,30 \\
\hline $\mathrm{B}_{\mathrm{C}}$ & 1250,00 \\
\hline
\end{tabular}

A Figura 5 apresenta a comparação entre os resultados numéricos obtidos pelo programa implementado e os apresentados por Proença, Papa e Maier (1991) para os deslocamentos do centro da viga parede.

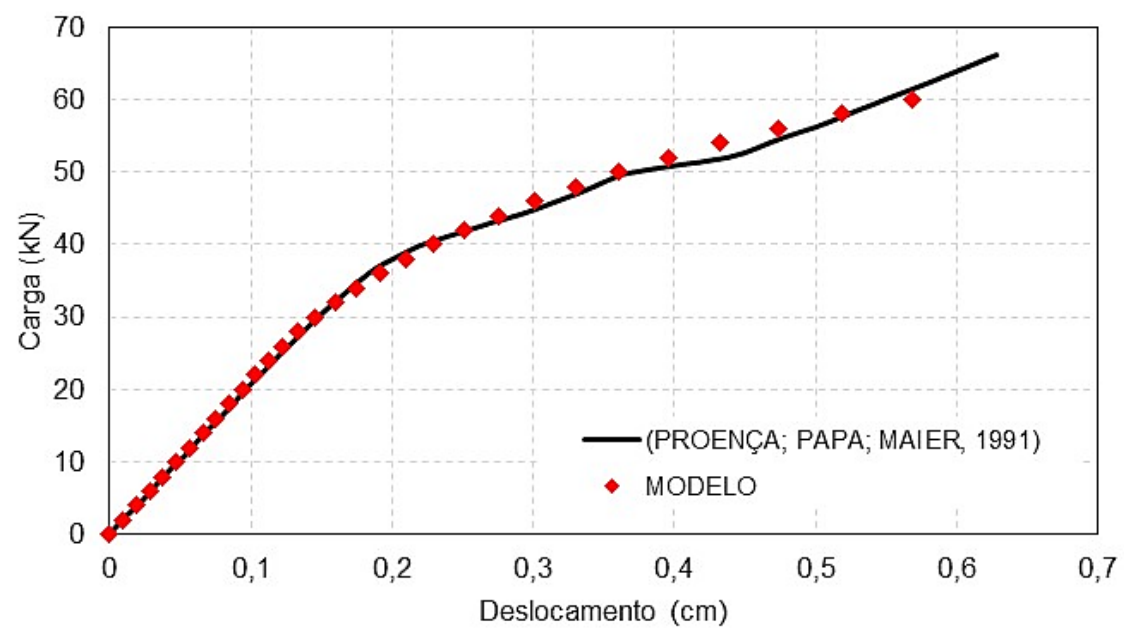

Figura 5: Carga versus deslocamento obtido na modelagem.

Observa-se que os resultados obtidos com o código implementado são semelhantes aos apresentados por Proença, Papa e Maier (1991), demonstrando que o mesmo obtém coerentemente os deslocamentos da viga tanto no regime elástico-linear quanto no não-linear. O mesmo demonstra ainda a eficiência do modelo de dano de Mazars quanto a representação da não-linearidade física do material. 


\subsection{Validação do modelo de formação dos produtos de corrosão}

A fim de validar e verificar se a formulação implementada para representar a formação dos produtos de corrosão, modelouse a expansão imposta pela corrosão. $\mathrm{O}$ elemento consiste em um cilindro de concreto contendo uma barra de aço centralizada. Na Figura 6(a) é apresentado elemento de concreto e na Figura 6(b) um esquema referente às condições de exposição ao $\mathrm{CO}_{2}$.

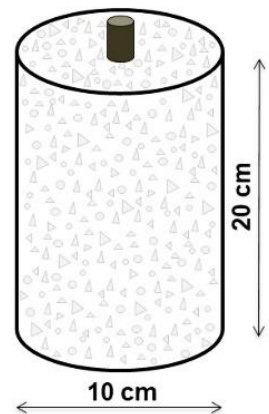

(a)

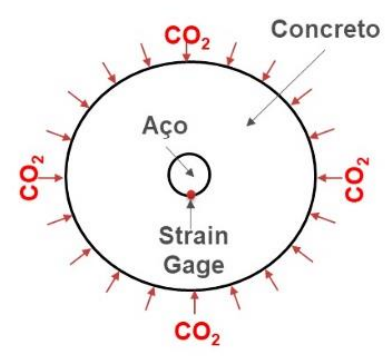

(b)

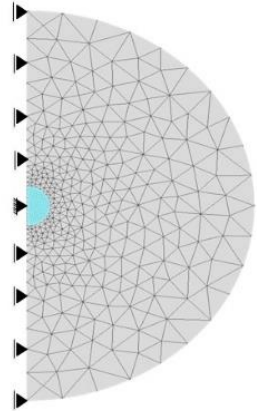

(c)

Figura 6: Detalhamento: (a) elemento; (b) exposição ao $\mathrm{CO}_{2}$ e (c) malha e condições de contorno.

Para a imposição da corrosão, considerou-se uma corrente uniforme ao longo do tempo, considerando a mesma com densidade de $500 \mu \mathrm{A} / \mathrm{cm}^{2}$ (valor utilizado em ensaios de corrosão acelerada). O diâmetro integro da armadura é de 10 $\mathrm{mm}$, a densidade do aço é de $7800 \mathrm{~kg} / \mathrm{m}^{3}$ e a proporção entre a densidade do aço e dos produtos de corrosão é de 4,50.

A discretização da malha do cilindro foi feita com 3265 nós e 706 elementos triangulares com ordem de aproximação cúbica para a representação da matriz de concreto, e com 422 elementos triangulares com ordem de aproximação cúbica para a representação da armadura, conforme Figura 6(c). Vale ressaltar que apenas metade do elemento foi modelado, devido à simetria existente no problema. Quanto às propriedades dos materiais, o concreto possuí módulo de elasticidade de $3497,00 \mathrm{kN} / \mathrm{cm}^{2}$, resistência à compressão de $4,00 \mathrm{kN} / \mathrm{cm}^{2}$, resistência à tração de $0,29 \mathrm{kN} / \mathrm{cm}^{2}$ e coeficiente de Poisson de 0,2 . Já o aço, possui módulo de elasticidade de $21000,00 \mathrm{kN} / \mathrm{cm}^{2}$ e resistência à tração de $50,0 \mathrm{kN} / \mathrm{cm}^{2}$. O modelo constitutivo empregado é o de Saint-Venant-Kirchhoff para materiais elástico-linear.

A Figura 7 apresenta a comparação entre os resultados numéricos (modelo proposto) e experimentais (NGUYEN et al., 2007) para o deslocamento vertical da interface aço/concreto (ponto contendo o Strain Gage), conforme Figura 6(b).

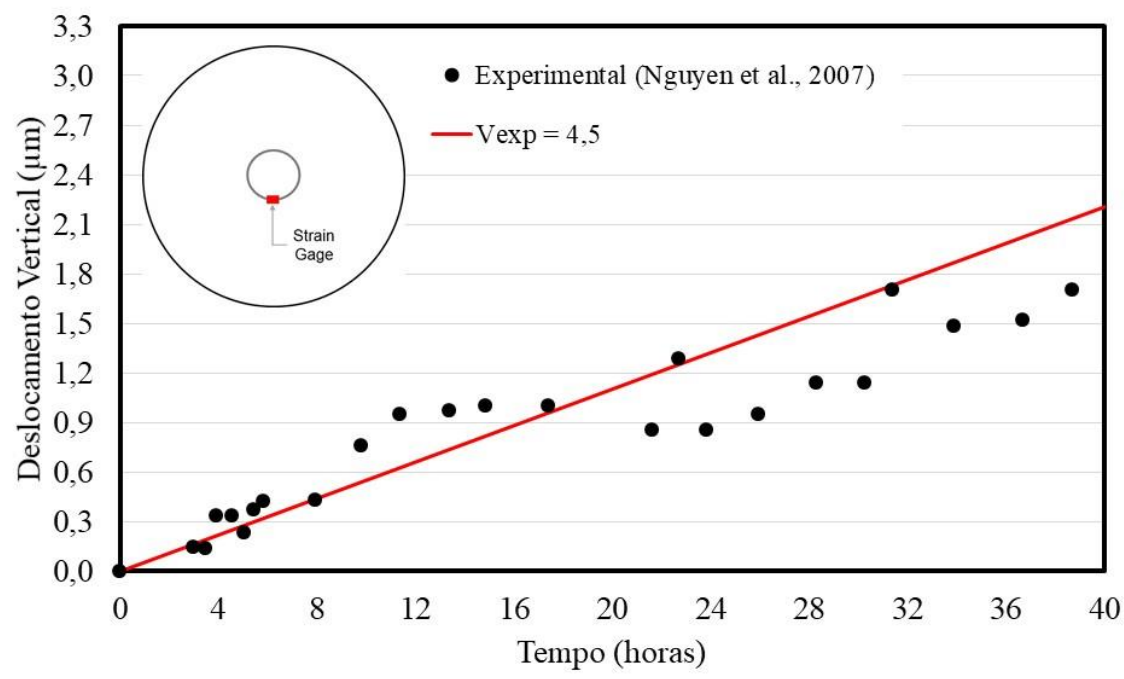

Figura 7: Deslocamento vertical da interface aço/concreto no tempo.

Na Figura 8 são apresentados os campos de deslocamento (vertical e horizontal), tensão (horizontal) e deformação principal do cilindro após 40 horas de exposição ao agente agressivo. 

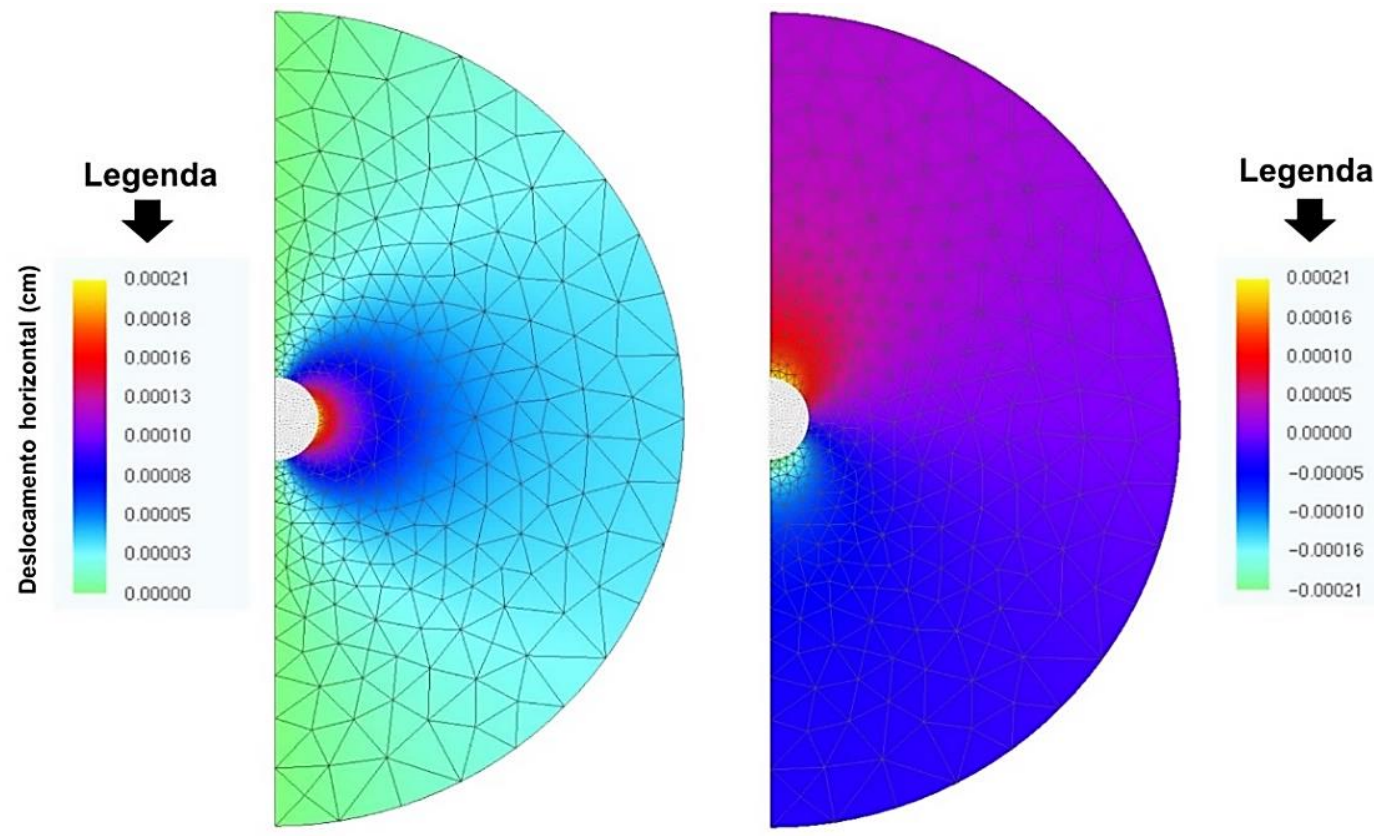

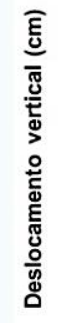
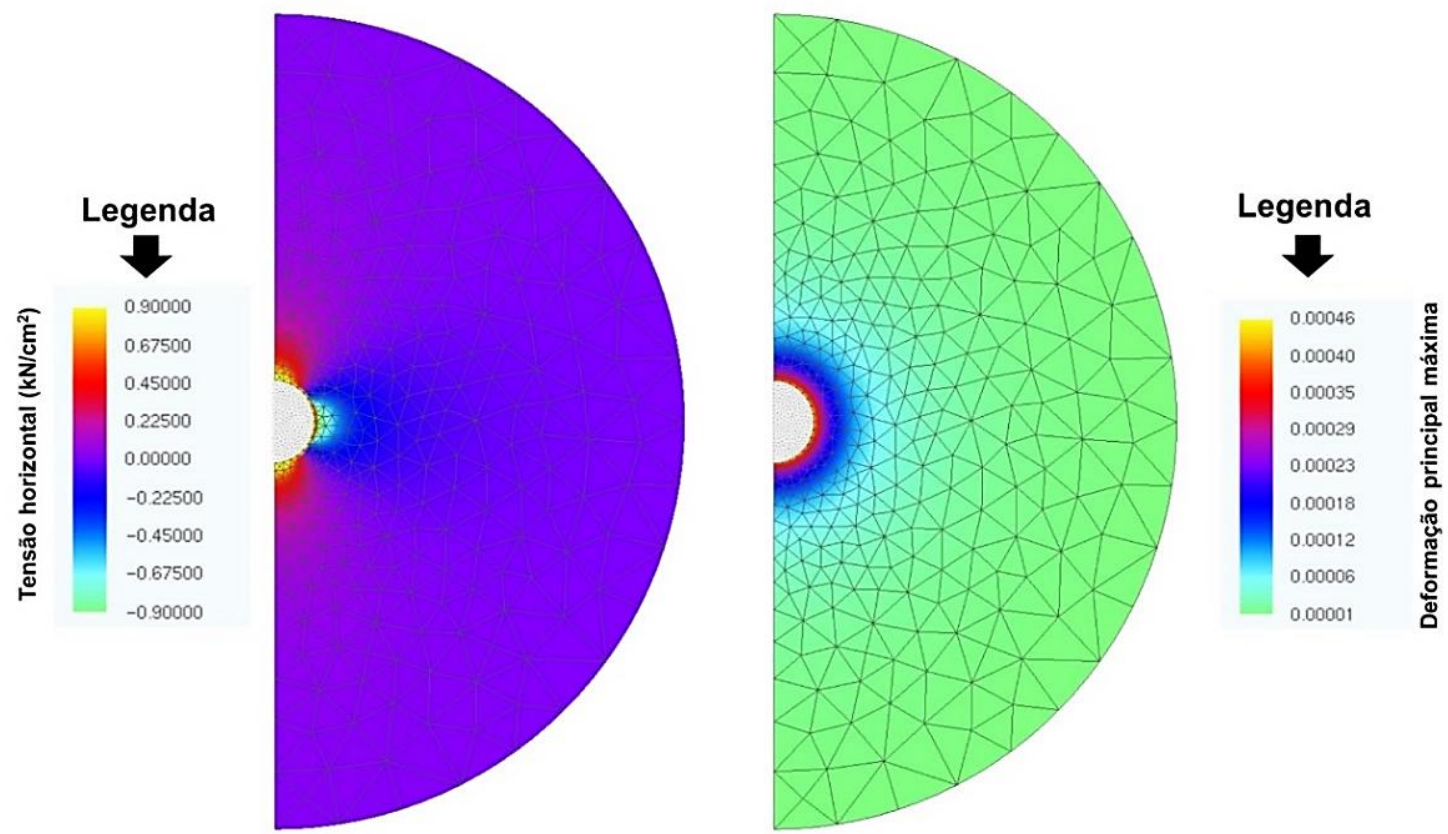

Figura 8: Deslocamentos, tensão horizontal e deformação principal após 40 horas de exposição.

Portanto, observa-se pelas Figuras 7 e 8 que o modelo representa de forma coerente o campo de deslocamento do elemento modelado quando considerado o regime elástico-linear.

\subsection{Aplicação e análise do modelo desenvolvido}

A fim de demonstrar a aplicabilidade do modelo proposto, modelou-se a expansão de um elemento de concreto armado devido à degradação das armaduras por corrosão. O elemento analisado consiste em uma viga retangular de concreto armado de $200 \mathrm{~cm}$ de comprimento, cuja seção transversal de 10x10 cm é mostrada na Figura 9(a). Na Figura 9(b) apresenta-se um esquema referente às condições de exposição do elemento de concreto ao agente agressivo, o $\mathrm{CO}_{2}$.

Para a modelagem do elemento decidiu-se por representar apenas 1/4 da seção transversal, uma vez que a seção transversal é bissimétrica. A discretização da malha de elementos finitos da viga de concreto armado foi feita com 4849 nós e 1064 elementos triangulares com ordem de aproximação cúbica para a representação da matriz de concreto e com 825 elementos triangulares com ordem de aproximação cúbica para a representação da armadura, conforme mostra a Figura 9(c). 


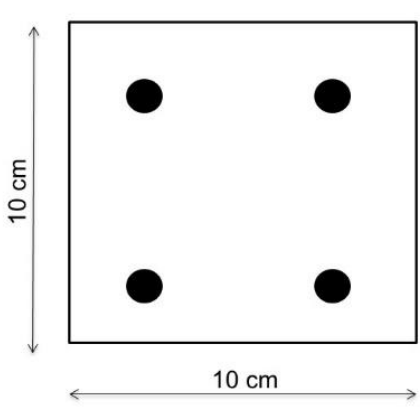

(a)

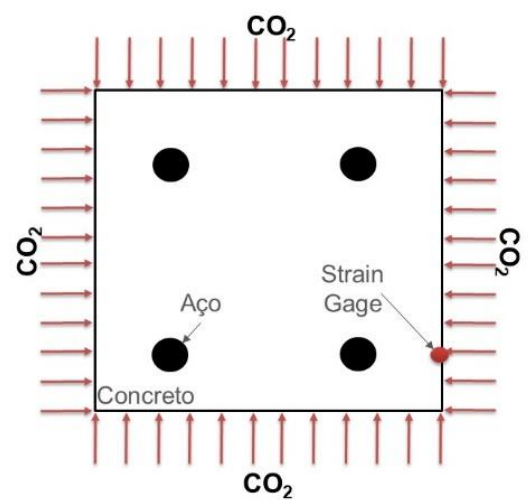

(b)

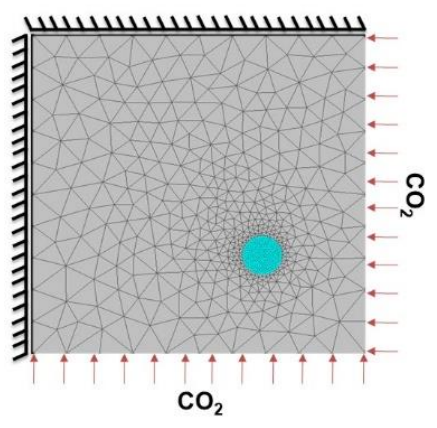

(c)

Figura 9: Detalhamento da seção da viga: (a) dimensões; (b) exposição ao $\mathrm{CO}_{2}$ e (c)condições de contorno.

Para a imposição da corrosão, considerou-se uma corrente uniforme ao longo do tempo, considerando a mesma com densidade de $100 \mu \mathrm{A} / \mathrm{cm}^{2}$. O diâmetro integro da armadura é de $19 \mathrm{~mm}$, a densidade do aço é de $7800 \mathrm{~kg} / \mathrm{m}^{3}$ e a proporção entre a densidade do aço e dos produtos de corrosão é de 4,50 .

O tempo de análise foi dado em função da danificação do elemento. Para este exemplo, a análise da expansão é finalizada quando o dano na matriz do concreto alcança 0,9 .

Os dados referentes às propriedades dos materiais são apresentados na Tabela 2(a), enquanto que na Tabela 2(b) apresentamse os dados referentes ao dano.

Tabela 2(a) - Propriedade dos materiais.

\begin{tabular}{c|c|c}
\hline Material & Propriedade & Valor \\
\hline \multirow{3}{*}{$\begin{array}{c}\text { Concreto } \\
(\mathrm{C} 25)\end{array}$} & Módulo de elasticidade $\left(\mathrm{kN} / \mathrm{cm}^{2}\right)$ & 3000,00 \\
\cline { 2 - 3 } & Coeficiente de Poisson & 0,20 \\
\cline { 2 - 3 } & Resistência à compressão $\left(\mathrm{kN} / \mathrm{cm}^{2}\right)$ & 2,50 \\
\cline { 2 - 3 } & Resistência à tração $\left(\mathrm{kN} / \mathrm{cm}^{2}\right)$ & 0,17 \\
\hline $\begin{array}{c}\text { Aço } \\
(\mathrm{CA}-50)\end{array}$ & Módulo de elasticidade $\left(\mathrm{kN} / \mathrm{cm}^{2}\right)$ & 21000,00 \\
\cline { 2 - 3 } & Resistência à tração $\left(\mathrm{kN} / \mathrm{cm}^{2}\right)$ & 50,00 \\
\hline
\end{tabular}

Tabela 2(b) - Dados referentes ao dano.

\begin{tabular}{c|c}
\hline Propriedade & Valor \\
\hline$\varepsilon \mathrm{d} 0$ & $7,0 \mathrm{E}-5$ \\
\hline $\mathrm{A}_{\mathrm{T}}$ & 0,995 \\
\hline $\mathrm{B}_{\mathrm{T}}$ & 8000,00 \\
\hline $\mathrm{A}_{\mathrm{C}}$ & 1,13 \\
\hline $\mathrm{B}_{\mathrm{C}}$ & 1650,00 \\
\hline
\end{tabular}

Apresenta-se na Figura 10 o avanço do deslocamento horizontal no ponto de análise, considerando o modelo com e sem dano.

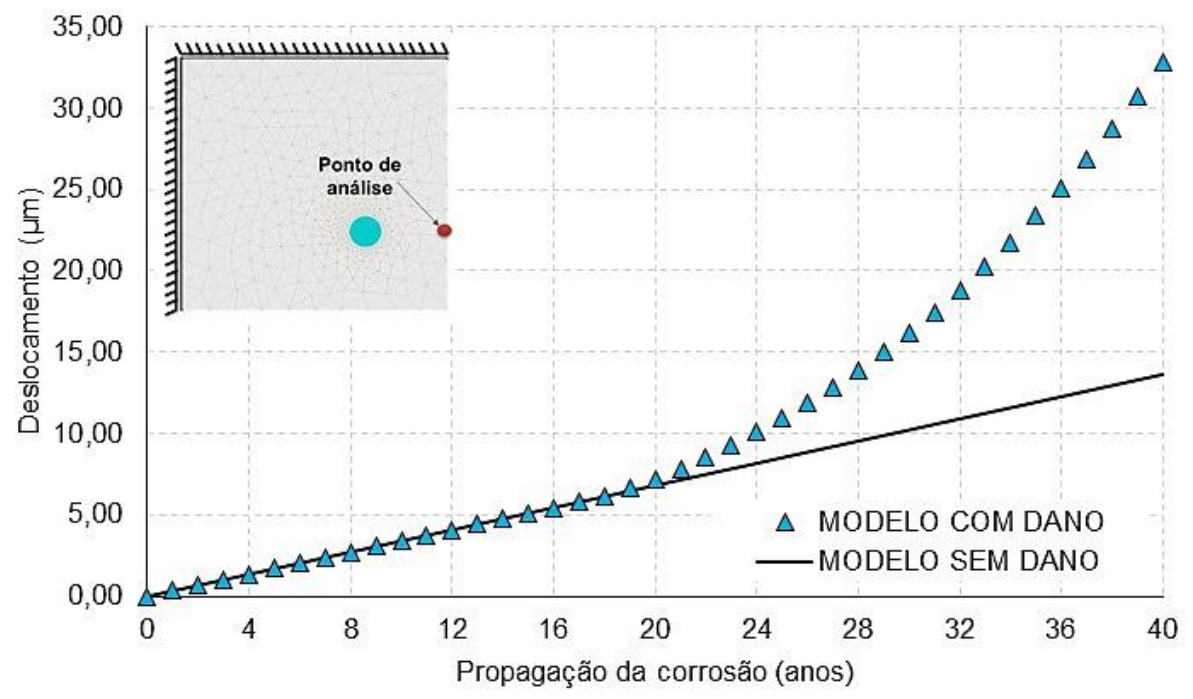

Figura 10: Deslocamento horizontal do ponto de análise. 
Observa-se que os deslocamentos são superiores quando considerada a formulação de dano, ou seja, quando considerada a nãolinearidade física do material. É possível notar ainda que os deslocamentos crescem exponencialmente à medida em que a corrosão progride.

Na Figura 11 são apresentados os campos de deslocamento (vertical e horizontal) e o estado de danificação do elemento de concreto para um tempo de progressão da corrosão de 19 anos (último passo de tempo em que não há dano), 20 (primeiro passo de tempo com dano) e 25 anos (5 anos após iniciar a danificação do material). Já na Figura 12, é apresentada a evolução do estado de danificação da viga de concreto armado ao longo do tempo.
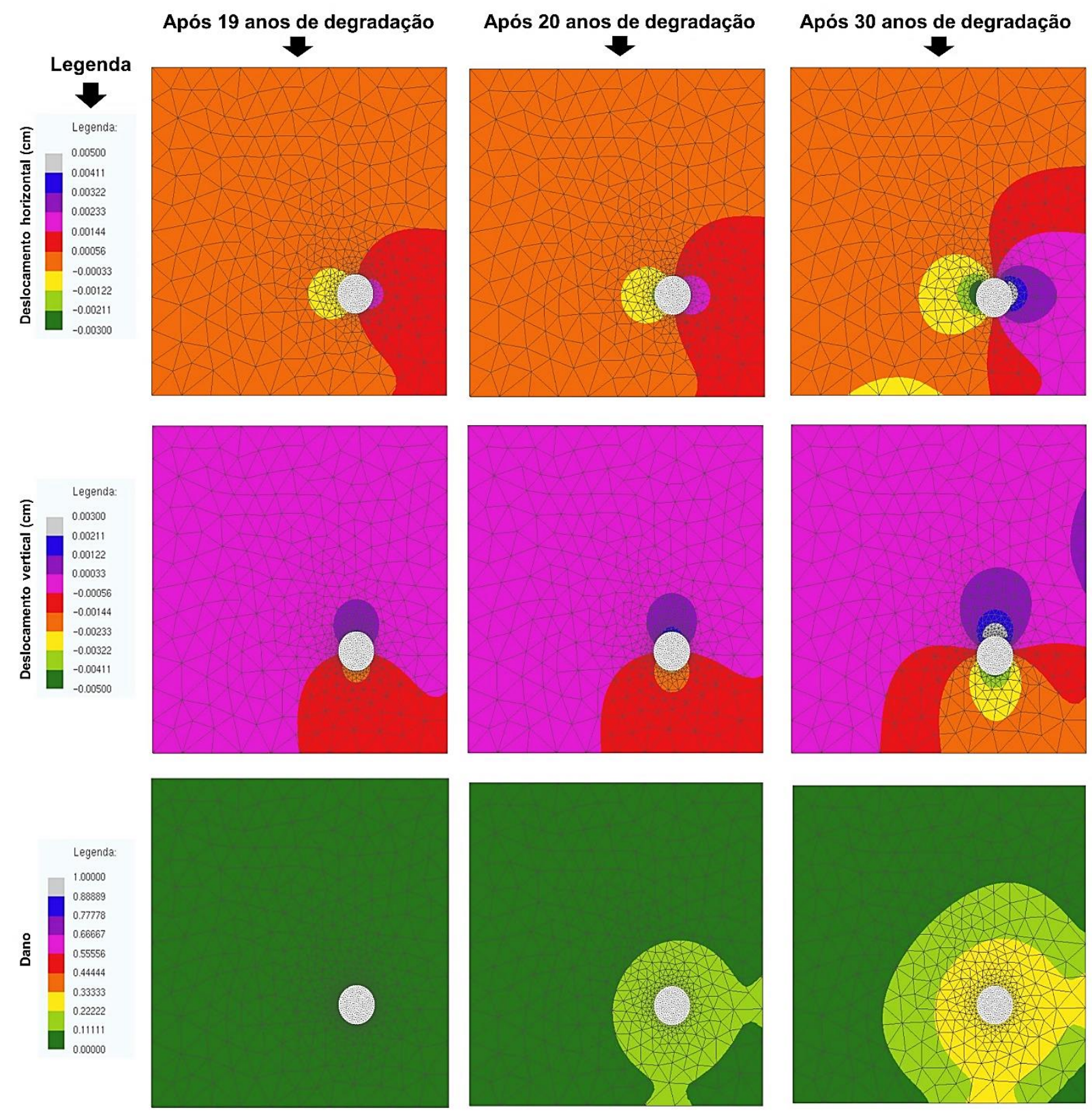

Figura 11: Deslocamentos e danificação do elemento de concreto aos 19, 20 e 30 anos.

Observa-se, neste caso, que os deslocamentos são mais expressivos para as faces da viga que estão mais próximas da armadura em degradação. Assim, se considerada a viga com sua seção transversal total, terimos maiores deslocamentos nas quatro extremidades, e pela simetria da peça, os deslocamentos experimentados pelo concreto de cobrimento teria módulo semelhante. Observa-se ainda, que para regiões distantes das armaduras, pouco, ou quase nenhum deslocamento é visto, isso ocorre devido às reações contrárias que surgem internamente no concreto, fazendo com que o mesmo passa a trabalhar para evitar as deformações impostas pelo surgimento dos produtos de corrosão. 


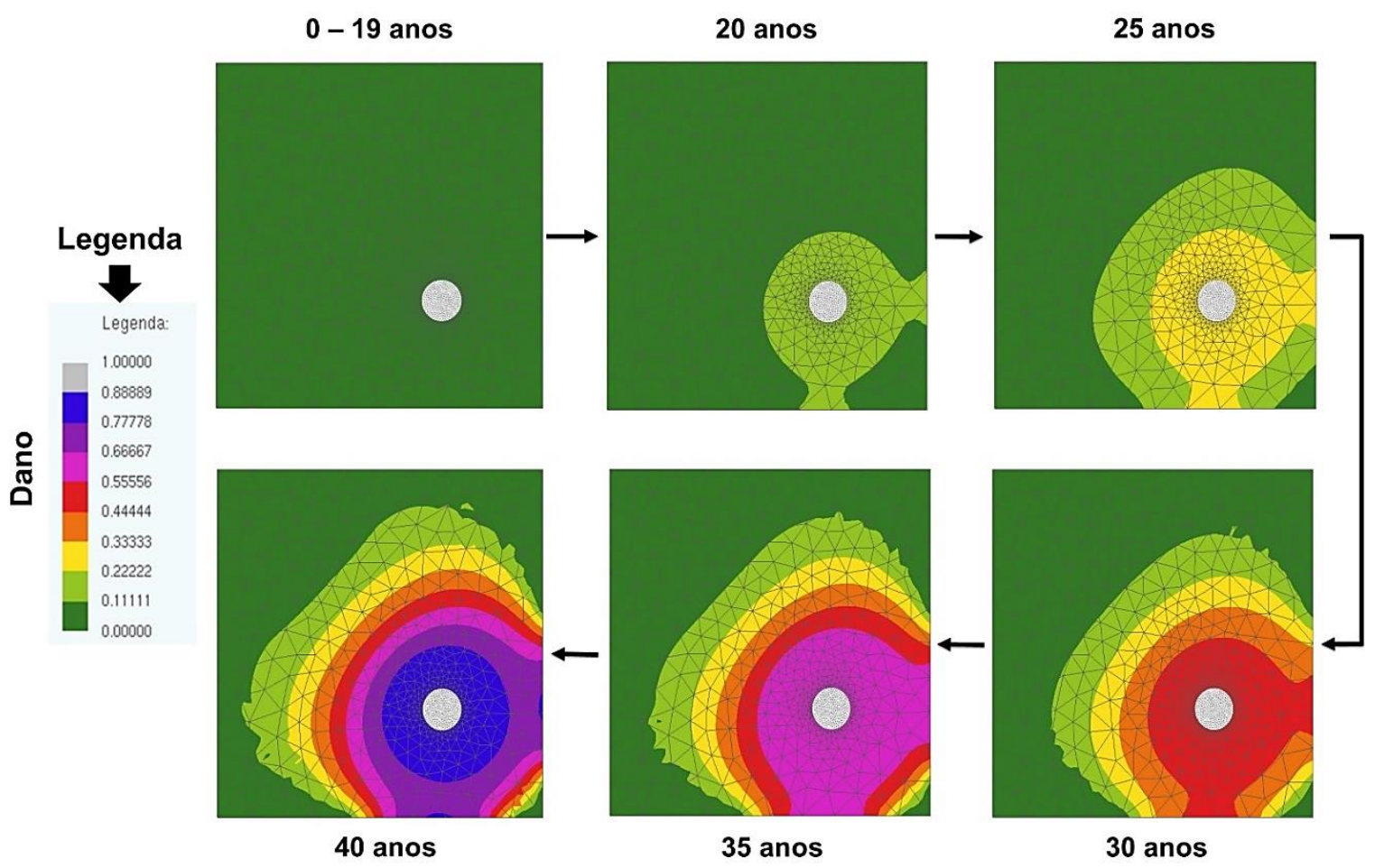

Figura 12: Avanço do dano em função do tempo de progressão da corrosão.

Por fim, observa-se pela Figura 12 que o dano evolui de forma progressiva, concomitantemente, à formação dos produtos de corrosão. Ademais, nota-se que o concreto da região da interface aço/concreto entra em estado total de danificação logo após a despassivação e progressão da corrosão, e que, após 20 anos do início da progressão da corrosão (40 anos, ao todo), o concreto de cobrimento já está totalmente danificado, demonstrando que provavelmente, já ocorreu a fissuração e lascamento do concreto de cobrimento, se não considerada nenhuma atividade de recuperação.

\section{CONSIDERAÇÕES FINAIS}

Diante dos resultados obtidos com o código desenvolvido, pode-se verificar que:

- O modelo de dano de Mazars encontra-se corretamente implementado e acoplado ao MEFP, possibilitando assim, representar coerentemente a não-linearidade física do material;

- Os resultados encontrados demonstram a eficiência da formulação implementada frente à análise estrutural de materiais compósitos, por exemplo, o concreto armado;

- Os resultados apontam a aplicabilidade do modelo quanto à representação da expansão do concreto decorrente da corrosão;

- O modelo desenvolvido descreve coerentemente as regiões que mais se danificam, quando do surgimento de produtos de corrosão, e que a depender da região (considerando a seção transversal do elemento de concreto), o elemento de concreto fissurar rapidamente e prematuramente;

Ademais, destaca-se que por mais que o código não represente a formação e a progressão das fissuras, a utilização do modelo de dano de Mazars possibilitou a análise das regiões do concreto que mais são danificadas à medida em que os produtos de corrosão são formados internamente ao concreto. Assim, mostrou-se que o código desenvolvido pode ser usado para a análise da degradação de peças estruturas de concreto armado, quando estás estão submetidas à degradação por corrosão uniforme.

\section{AGRADECIMENTOS}

Os autores agradecem ao Conselho Nacional de Desenvolvimento Científico e Tecnológico (CNPq) pelo fomento fornecido à pesquisa (CNPq 141078/2018 e CNPq 310564/2018-2). Este estudo também foi financiado pela Cordenação de Aperfeiçoamente de Pessoal de Nível Superior (CAPES código 001). 


\section{REFERÊNCIAS}

ANDRADE, C. Manual para diagnóstico de obras deterioradas por corrosão de armaduras. Trad. De Antonio Carmona e Paulo Helene. São Paulo, Pini, 1992.

BAKKER R. M. F. Initiation period. In: Schiess P. Corrosion of steel in concrete, London, Chapman and Hall, cap. 3, pp. 22-55, 1988.

BROOMFIELD, J. P. Corrosion of steel in concrete: understanding, investigation and repair. 2. Ed. New York, Taylor \& Francis, pp. 294, 2007.

CARMONA, A. F.; MAREGA, A. Retrospectiva da patologia no Brasil: Estudo Estatístico, in: Jornadas em Español y Portugués sobre Estructuras y Materiales, CEDEX, IETcc, (1988), pp. 325-348, 1988.

CODA H. B. Análise não linear geométrica de sólidos e estruturas: uma formulação posicional baseada no MEF, Volume II, Departamento de Estruturas, Escola de Engenharia de São Carlos, Universidade de São Paulo, São Carlos, 2003.

DAL MOLIN, D. C. C. Fissuras em estruturas de concreto armado: análise das manifestações típicas e levantamento de casos ocorridos no Estado do Rio Grande do Sul, Dissertação de Mestrado em Engenharia, Universidade Federal do Rio Grande do Sul, Porto Alegre, 1988.

FELIX, E. F. Modelagem da deformação do concreto armado devido à formação dos produtos de corrosão. Dissertação. Escola de Engenharia de São Carlos, Programa de Pós-Graduação em Engenharia de Estruturas, EESC/USP, São Carlos, 2018.

KARI, O. P.; PUTTONEN, J.; SKANTZ, E. Reactive transport modelling of long-term carbonation, Cement and Concrete Composites, v.52, pp. 42-53, 2014.

KIANI K.; SHODJA H. M. Prediction of the penetrated rust into the microcracks of concrete caused by reinforcement corrosion. Applied Mathematical Modelling, v. 35, n. 5, p. 2529-2543, 2011.

KÖLIÖ, A.; PAKKALA, T. A.; HOHTI, H.; LAUKKARINEN, A.; LAHDENSIVU,J.; MATTILA, J.; PENTTI, M. The corrosion rate in reinforced concrete facades exposed to outdoor environment, Materials and Structures, V.50, pp. 1-16, 2017.

MAZARS, J. Application de la mechanique de l'endommagement au comportamento non lineaire et à la rupture du béton de struture, Thèse de Doctorat d'etat, Université Paris 6, Paris, (1984).

NGUYEN, T. T. H.; BARY, B.; DE LARRARD, T. Coupled carbonation-rust formation damage modeling and simulation of steel corrosion in 3D mesoscale reinforced concrete. Cement and Concrete Research, v. 74, p. 95-107, 2015.

PACCOLA R. R.; CCODA, H. B. A direct FEM approach for particulate reinforced elastic solids. Composite Structures, v. 141, n. 1, p. 282-291, 2016.

POSSAN, E. Modelagem da carbonatação e previsão de vida útil de estruturas de concreto em ambiente urbano. Tese (Doutorado em Engenharia) - Escola de Engenharia, Programa de Pós-Graduação em Engenharia Civil, UFRGS, Porto Alegre, 2010.

PRONÇA, S. P. B. Simulação numérica do comportamento de elementos estruturais em concreto e argamassa armada pelo emprego de um modelo de dano. Revista Internacional de Métodos Numéricos para Cálculo y Diseño em Ingeniería, v. 8, p. 351360, 1992.

PROENÇA S. P. B; PAPA E.; MAIER G. Meccanica del danneggiamento di material e strutture: aplicazioni al calcestruzzo. Rapporto sul contrato di ricerca 90-91. Departimento di Ingegneria Strutturale. Politectico di Milano, 1991.

RAO, A. S.; LEPECH, M. D.; KIREMIDJIAN, A. S.; SUN, X. Y. Simplified structural deterioration model for reinforced concrete bridge piers under cyclic loading, Structure and Infrastructure Engineering, V.13, pp. 55-66, 2017.

TUUTTI, K. Corrosion of Steel in Concrete. Swedish Cement and Concrete Research Institute. Stockholm, Swedish, 1982. 30p., 1982.

VAL D. V., MELCHERS R. E. Reliability of deteriorating RC slab bridges, Journal of Structural Engineering 123, pp. 16381644, 1997. 